\title{
Two estrogen receptors expressed in the teleost fish, Sparus aurata: cDNA cloning, characterization and tissue distribution
}

\author{
S Socorro, D M Power, P-E Olsson ${ }^{1}$ and A V M Canario \\ Centro de Ciências do Mar, Universidade do Algarve, Campus de Gambelas, 8000 Faro, Portugal \\ ${ }^{1}$ Department of Cell and Molecular Biology, Division of Physiology, Umea University, 90187 Umea, Sweden \\ (Requests for offprints should be addressed to A V M Canario; Email: acanario@ualg.pt)
}

\begin{abstract}
Estrogen is an essential hormone for many reproductive and non-reproductive functions. The function of estrogen in the reproductive cycle of seabream (Sparus aurata), a protandrous hermaphrodite teleost fish, is complex but it is understood to be involved in sex inversion, a process that occurs in some individuals during the second reproductive season. Estrogen action is mediated by two estrogen receptor (ER) subtypes designated alpha and beta. As a step to understanding the mechanisms of estrogen action during natural and induced sex reversal in seabream, we have isolated two cDNAs encoding distinct forms of ER homologous to mammalian $\operatorname{ER} \alpha$ and $\operatorname{ER} \beta$. The seabream ER $\alpha$ clone (sbER $\alpha 1)$, which was truncated in the A/B domain, corresponded to a variant differing in five amino
\end{abstract}

acids from another recently cloned $\operatorname{sbER} \alpha$. The ER $\beta$ clone (sbER $\beta$ ) encoded a protein 559 amino acids long and showed only $40 \%$ identity to sbER $\alpha$. Northern blot analysis of liver and ovary mRNA indicated the presence of several transcripts of the two receptor subtypes. PCR analysis showed that the two receptors differed in their expression pattern. sbER $\alpha$ had a more restricted distribution, occurring mainly in testis, liver and heart, and $\operatorname{sbER} \beta$ was present in most tissues, being more abundant in ovary, testis, liver, intestine and kidney. The presence in seabream of two ERs with several ER transcripts and their pattern of distribution are consistent with the widespread effects of estrogen in different tissues.

Journal of Endocrinology (2000) 166, 293-306

\section{Introduction}

Estrogen is a steroid hormone essential in several aspects of reproduction throughout the vertebrates, and also has many non-reproductive roles better known in mammals. Estrogen action is mediated by nuclear receptors, the first estrogen receptor (ER) being cloned from human more than 10 years ago (Walter et al. 1985, Green et al. 19866) and was followed by the cloning of similar receptors from rat (Koike et al. 1987), chicken (Krust et al. 1986) and Xenopus (Weiler et al. 1987). More recently, a novel cDNA encoding a different estrogen receptor, ER $\beta$, has been cloned in rat (Kuiper et al. 1996), mouse (Tremblay et al. 1997) and human (Mosselman et al. 1996, Enmark et al. 1997, Ogawa et al. 1998b), and has raised new questions regarding the mechanism of action and physiology of the ERs.

The ER belongs to the nuclear receptor superfamily and is included in the steroid receptor subfamily (Laudet 1997). Steroid receptor proteins are divided into six functionally independent domains, termed A to F from the amino to carboxyl terminus (Krust et al. 1986). The $\mathrm{N}$-terminal region (domain $\mathrm{A} / \mathrm{B}$ ) has been demonstrated to have a cell-type and promoter specific transactivation function (AF-1) (Tora et al. 1989, Tzukerman et al. 1994). The central region (domain C or DNA-binding domain, DBD) is highly conserved among species and is responsible for DNA binding (Kumar et al. 1987). A nuclear localization signal, homologous to that of SV40 large antigen T, was identified in domain D (Picard et al. 1990). In the $\mathrm{C}$-terminal region, the $\mathrm{E}$ domain (or ligand-binding domain, LBD) is required for ligand binding (Kumar et al. 1987) and includes a ligand-dependent transactivation function (AF-2) (Danielian et al. 1992). The function of the $\mathrm{F}$ domain is not completely clear, but it is proposed to have a modulatory role that affects the agonist/antagonist effectiveness of antiestrogens and the transcriptional activity of the ligand-receptor complex in cells (Montano et al. 1995).

ER $\beta$ cDNAs encode a protein with high amino acid identity with the ER $\alpha$ protein, particularly in the DBD (96-97\%) and LBD (53-60\%) (Tremblay et al. 1997, Ogawa et al. 1998b). Ligand binding studies using proteins synthesized in vitro have indicated that most estrogenic and anti-estrogenic compounds bind both forms of ER with a similar affinity (Kuiper et al. 1996), but may have different mechanisms regulating transcriptional activity (Tremblay et al. 1997); the two different forms of ER can also 
dimerize and generate a functional unit (Pace et al. 1997). Important differences have been found in the tissue distribution and/or the relative levels of expression of ER $\beta$ and ER $\alpha$ mRNA. RT-PCR analysis of various rat tissues showed moderate to high expression of ER $\alpha$ in uterus, testis, pituitary, ovary, kidney, epididymis and adrenal gland, while ER $\beta$ was more abundantly expressed in prostate, ovary, lung, bladder, brain, bone, uterus and testis (Kuiper et al. 1997). Besides the different pattern of tissue expression within the same organ, differential expression of both forms of ER has been noted in different cell types (Byers et al. 1997, Osterlund et al. 1998, Shughrue et al. 1998). This differential expression suggests tissue-specific roles for each ER subtype and that different effects may be mediated by homodimers or heterodimers of the two receptors. Studies with the ER-knockout mice $\alpha E R K O, \beta E R K O$ and $\alpha \beta E R K O$ indicate that some biological functions of estrogen require the presence of both receptors (Krege et al. 1998, Couse \& Korach 1999, Couse et al. 1999).

The ER has been cloned in several teleost fish, including rainbow trout, Oncorhynchus mykiss (Pakdel et al. 1990), killifish, Oryzias spp. (accession number D28954), tilapia, Oreochromis aureus (Tan et al. 1995), channel catfish, Ictalurus punctatus (Xia et al. 1999), Japanese eel, Anguilla japonica (Todo et al. 1996), red seabream, Chrysophrys major (Touhata et al. 1998), gilthead seabream, Sparus aurata (Munoz-Cueto et al. 1999) and goldfish, Carassius auratus (Tchoudakova et al. 1999). All fish ERs, excluding the Japanese eel and goldfish, are more related to ER $\alpha$.

The function of estrogen in the reproductive cycle of seabream, a protandrous hermaphrodite teleost fish, is complex. During the first reproductive cycle this fish develops functional testis although administration of estrogen causes testicular regression (Condeça \& Canario 1999) and eventually the development of functional ovaries (Happe \& Zohar 1988). As a step to understanding the mechanisms of estrogen action during natural and induced sex reversal in seabream, we have isolated two cDNAs encoding distinct forms of ER homologous to mammalian $E R \alpha$ and $E R \beta$ respectively and studied their tissue expression.

\section{Materials and Methods}

\section{Production of an ER $c D N A$ probe}

Total RNA was extracted from estradiol $\left(\mathrm{E}_{2}\right)$-stimulated liver by an adaptation of the acid guanidinum thiocyanatephenol-chloroform extraction method (Chomczynski \& Sacchi 1987). Liver total RNA (5 $\mu \mathrm{g})$ was reversetranscribed using Moloney murine leukaemia virus (MMLV)-RT (Gibco BRL, Barcelona, Spain) and oligo(dT) ${ }_{12-18}$ primer (Pharmacia Biotech, Lisbon, Portugal) in a final volume of $30 \mu \mathrm{l}$. Two degenerate PCR primers were designed to amplify a fragment of seabream
ER cDNA which spanned conserved regions in the DNA and hormone-binding domains: forward primer, 5'-TAYGGNKTKTGGTCNTGYGA-3' (YGVWSCE) and reverse primer $5^{\prime}$-TGYTCCATKCCKTTRTT RCT-3' (SNKGMEH). PCR amplification was carried out with $5 \mu$ l of synthesized cDNA using $2.5 \mathrm{U}$ of Taq polymerase (Gibco, BRL) and 50 pmol of each degenerate primer. PCR cycling $94{ }^{\circ} \mathrm{C}, 1 \mathrm{~min} 15 \mathrm{~s} ; 50{ }^{\circ} \mathrm{C}, 2 \mathrm{~min}$; $72{ }^{\circ} \mathrm{C}, 50 \mathrm{~s}$ was repeated 35 times, followed by a final 10 min extension at $72{ }^{\circ} \mathrm{C}$. A fragment of the predicted size $(1000 \mathrm{bp})$ was purified directly from the PCR reaction using Wizard PCR Preps DNA Purification System (Promega, Biocontec, Lisbon, Portugal), cloned into pGEM-T Easy Vector (Promega) and sequenced. This product (GenBank accession number AF 013104) was highly homologous to ER and was used as a probe to screen cDNA libraries of liver, pituitary and ovary of seabream.

\section{Construction and screening of $c \mathrm{DNA}$ libraries}

Three cDNA libraries were constructed in UNI-ZAP XR vector (Stratagene, Biocontec, Lisbon, Portugal) with reverse-transcribed cDNA of seabream $\mathrm{E}_{2}$-stimulated liver, pituitary and ovary obtained from $5 \mu \mathrm{g}$ of $\operatorname{poly}(\mathrm{A})^{+}$ RNA and using the UNI-ZAP XR cDNA synthesis kit (Stratagene) according to supplier's instructions. Screening was carried out under high stringency conditions. Duplicate membranes (Hybond-C, Amersham, Lisbon, Portugal) were hybridized with the $\left[{ }^{32} \mathrm{P}\right]-\alpha$-dCTP-labeled PCR product overnight at $65^{\circ} \mathrm{C}$ in a solution containing $6 \times$ SSC, $5 \times$ Denhart's, $0 \cdot 1 \%$ SDS and $0 \cdot 1 \mathrm{mg} / \mathrm{ml}$ transfer RNA. Stringency washes were carried out at $65^{\circ} \mathrm{C}$ with $0 \cdot 1 \times$ SSC containing $0 \cdot 1 \%$ SDS. Several positive clones were obtained after first round screening of $4 \times 10^{5}$ liver or pituitary phages. Positive clones isolated from each of these libraries were sequenced (Licor DNA4200 sequencer, MWG Biotech-UK, UK) and shown to have identical sequence where they overlapped. The largest clone (Z22) of $3.4 \mathrm{~kb}$, isolated from the liver library, was used for further analysis. Screening $2 \times 10^{5}$ phages of the ovary library with the same probe, yielded only one positive clone (Q45) of $2 \cdot 2 \mathrm{~kb}$ which was isolated and characterized.

\section{In vitro transcription and translation}

The complete Q45 cDNA inserted in the phagemid Bluescript $\mathrm{SK}(+/-)$ was translated in vitro in a rabbit reticulocyte lysate assay with $20 \mu \mathrm{Ci}$ of $\mathrm{L}-\left[{ }^{35} \mathrm{~S}\right]$ methionine (Amersham). Reactions were performed using the 'TNT T3 Quick coupled Transcription/Translation System' following suppliers instructions (Promega). Translation products $(5 \mu \mathrm{l})$ were analyzed by sodium dodecyl sulfate-polyacrylamide gel electrophoresis under reducing conditions. 
Sequence analysis

DNA sequences were analyzed using BLASTN and BLASTX (version 2.0, National Center for Biotechnology Information, Altschul et al. 1997) for database search, DNASIS version 5. 0 for deduction of amino acid sequence of cDNA, ClustalX for multiple sequence alignment (version 1. 64b, Thompson et al. 1997), GeneDoc for sequence editing (Nicholas et al. 1997) and Phyllip (version 3.5c, Felsenstein 1989) for phylogenetic analysis. The following ER sequences were used for multiple sequence alignment and phylogenetic tree analysis: seabream clones isolated in the present study, accession numbers AF136979 (clone Z22) and AF136980 (clone Q45), seabream clone isolated by Munoz-Cueto et al. (1999, sbER a2), red seabream (rsER, Touhata et al. 1998), tilapia (tER, Tan et al. 1995), Nile tilapia (ntER $\alpha$, accession number U75604), killifish (kER, accession number D28954), short and long forms of rainbow trout $\mathrm{ER} \alpha$ (rtER $\alpha$ s and rtER $\alpha$ l, accession numbers AJ242741 and AJ242740 respectively), Japanese eel (eER, Todo et al. 1996), channel catfish (ccER, Xia et al. 1999), zebra finch (zfER, Jacobs et al. 1996), chicken (cER, Krust et al. 1986), Xenopus (xER, Weiler et al. 1987), rat ER $\alpha(\mathrm{rER} \alpha$, Koike et al. 1987), sheep (sER, Madigou et al. 1996), human ER $\alpha$ (hER $\alpha$, Green et al. 1986b), Nile tilapia ER $\beta$ (ntER $\beta$, accession number U75605), goldfish ER $\beta$ (gfER $\beta$, Tchoudakova et al. 1999), rat $\operatorname{ER} \beta$ (rER $\beta$, accession number AJ002602), Japanese quail ER $\beta$ (jqER $\beta$, Lakaye et al. 1998), mouse $\operatorname{ER} \beta$ (mER $\beta$, Tremblay et al. 1997 and accession number AF067422) and human $\operatorname{ER} \beta$ (hER $\beta$, Ogawa et al. 1998b).

\section{Northern blot analysis}

Poly $(\mathrm{A})^{+}$mRNA (5 $\left.\mu \mathrm{g}\right)$ of adult seabream liver and ovary were separated on a $1 \%$ formaldehyde-agarose gel and transferred to Hybond-N (Amersham). The entire Q45 cDNA and a $341 \mathrm{bp}$ fragment of clone Z22 obtained by PCR (see details below) were radiolabeled with $\left[{ }^{32} \mathrm{P}\right]-\alpha$-dCTP (NEN, Zaventem, Belgium) using random priming (Redi-Prime, Amersham). Pre-hybridization was conducted for $3 \mathrm{~h}$ at $42{ }^{\circ} \mathrm{C}$ in $50 \%$ formamide, $5 \times \mathrm{SSC}$, $5 \times$ Denhardt's solution, $50 \mathrm{mM}$ sodium phosphate, $0 \cdot 1 \%$ SDS and $50 \mu \mathrm{g} / \mathrm{ml}$ calf thymus DNA. Hybridization was carried out overnight at the same temperature in an identical solution to which $2 \times 10^{6}$ c.p.m. per $\mathrm{ml}$ of denatured probe had been added. Stringency washes were carried out at $60{ }^{\circ} \mathrm{C}$ with $1 \times \mathrm{SSC}, 0 \cdot 1 \%$ SDS five times during $10 \mathrm{~min}$, and membranes were exposed to Biomax-MS film (Kodak, NY, USA) for an appropriate time.

\section{$R T$-PCR analysis}

Total RNA was extracted from ovary, testis, liver, brain, heart, bone, kidney, intestine, gills, muscle and skin of adult seabream using the Tri reagent protocol (SigmaAldrich Co., Madrid, Spain) and was reverse-transcribed as described above. PCR reactions were carried out using $5 \mu \mathrm{l}$ of the synthesized cDNA. A $341 \mathrm{bp}$ of clone Z22 and a $413 \mathrm{bp}$ fragment of clone Q45 were amplified (35 cycles $\left.94{ }^{\circ} \mathrm{C}, 1 \mathrm{~min} ; 65^{\circ} \mathrm{C}, 1 \mathrm{~min} 30 \mathrm{~s} ; 72{ }^{\circ} \mathrm{C}, 1 \mathrm{~min}\right)$ using primers specific for each clone. Seabream homologous primers were designed to the highly variable $\mathrm{N}$-terminal region of each receptor using Primer Premier software (version 4·1, Premier Biosoft International, Palo Alto, CA, USA) (Figs 1 and 2) to ensure receptor-specific PCR products. A fragment of $220 \mathrm{bp}$ of the seabream $\beta$-actin was also amplified from the same volume of synthesized cDNA $\left(35\right.$ cycles $94{ }^{\circ} \mathrm{C}, 1 \mathrm{~min} ; 50^{\circ} \mathrm{C}, 1 \min 30 \mathrm{~s} ; 72{ }^{\circ} \mathrm{C}$, $50 \mathrm{~s}$ ) using the oligonucleotides $5^{\prime}$ TTCCTCGGTATG GAGTCC3' and 5'GGACAGGGAGGCCAGGA3' (Santos et al. 1997).

\section{Results}

\section{Cloning of two estrogen receptors}

RT-PCR of sea bream liver using the degenerate primers to the DBD and LBD, amplified a $1000 \mathrm{bp}$ fragment of CDNA which was isolated, cloned and sequenced. A search in the GenBank database indicated highest sequence identity to the majority of identified fish ERs and to other isolated vertebrate ERs (data not shown). This fragment was used to screen seabream cDNA libraries. Liver and pituitary cDNA library screening yielded several clones identical with the probe in the corresponding region. The complete sequence of the largest clone (Z22-isolated from the liver library), $3461 \mathrm{bp}$ in length, was determined. Clone Z22 contained one ATG codon at nucleotide 92, but since it lacked an upstream in-frame stop codon and had a shorter A/B domain it was presumed truncated at the $5^{\prime}$ end. The $3^{\prime}$-untranslated region (UTR), including a poly $(\mathrm{A})^{+}$tail, was $1826 \mathrm{bp}$ long. The nucleotide sequence and deduced amino acid sequence of clone $\mathrm{Z} 22$ is shown in Fig. 1.

Ovary library screening yielded one positive clone (Q45), which was $2183 \mathrm{bp}$ in length and had a different sequence from the probe (data not shown). Its nucleotide sequence is given in Fig. 2. Clone Q45 contained an in-frame ATG codon at nucleotide 286 , preceded by an in-frame stop codon at nucleotide 232, suggesting it to be the likely start codon. A second downstream potential ATG start codon was located at nucleotide 384. Q45 contained a 285 bp 5'-UTR, followed by an open reading frame (ORF) with $1679 \mathrm{bp}$ and a 216 bp 3'-UTR including a poly $(\mathrm{A})^{+}$tail. The encoded protein was deduced to be 559 amino acids long and this was confirmed by in vitro translation of clone Q45 using the rabbit reticulocyte lysate assay. Analysis of the translation products on SDS-PAGE, $12 \%$ acrylamide gel revealed a protein doublet migrating 
1 CCA CCC TCA GAT GGG AGC CTT CAG TCC CTG GGC AGC GGG CCC AAC AGC CCT CTA GTG TTT GTG CCC TCC AGC CCC CAT CTC AGC

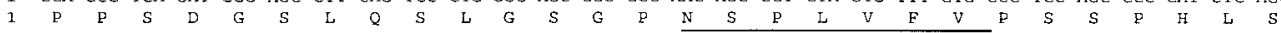

85 CCC TTC ATG CAC CCG CCC ACC CAC CAC TAT CTG GAA ACC ACC TCA ACA CCC ATC TAC AGG TCT AGT GTC CCA TCC AGT CAG CAT

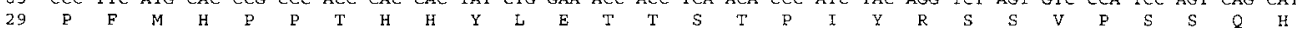

169 TCA GTT TCA AGA GAG GAC CAG TGT GGC ACC AGT GAC GAC TCA TAC AGT GTG GGG GAG TCA GGG GCT GGA GCG GGA GCC GCT GGG

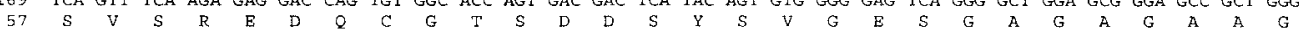

253 TTT GAG ATG GCC AAA GAG ATG CGT TTC TET GCC GTG TGC AGC GAT TAT GCC TCT GGG TAC CAT TAC GGG GTG TGG TCC TGT GAG

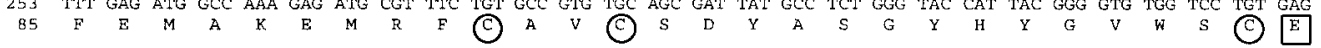

337 GGC TGC AAG GCC TTC TTT Aag AGg AGC ATA CAG GGT CAC Aat GAC TAT ATG TGC CCA GCA ACC AAT CAG TGT ACT ATT GAC AGG

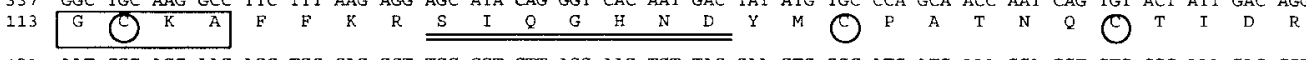

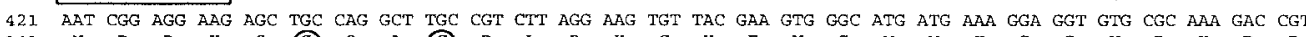

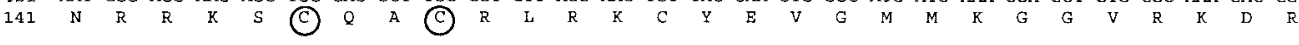

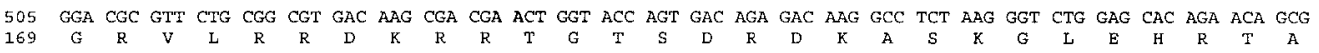
589 CCC CCT CAG GAC AGG AGG AAA CAC ATC AGC AGC AGT GCT GGT GGT GGA GGA GGA AAG TCA TCG GTG ATC AGC ATG CCT CCT GAC $\begin{array}{llllllllllllllllllllllllllllllllll}197 & P & P & Q & D & R & R & K & H & I & S & S & S & A & G & G & G & G & G & K & S & S & V & I & S & M & P & P & D\end{array}$

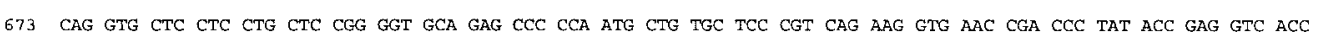

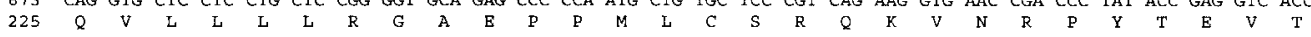
757 GTG ATG ACC CTG CTC ACC AGC ATG GCT GAT AAG GAG CTA GTC CAC ATG ATC GCA TGG GCC AAG AAG CTT CCA GGT TTC CTA CAG $\begin{array}{llllllllllllllllllllllllllllllllll}253 & \text { V } & \text { M } & \text { T } & \text { L } & \text { L } & \text { T } & \text { S } & \text { M } & \text { A } & \text { D } & \text { K } & \text { B } & \text { L } & \text { V } & \text { H } & \text { M } & \text { I } & \text { A } & \text { W } & \text { A } & \text { K } & \text { K } & \text { I } & \text { P } & \text { G } & \text { F } & \text { L } & Q & Q\end{array}$ 841 CTG TCC CTC CAC GAC CAA GTG CAG CTG CTG GAG AGC TCG TGG CTG GAG GTG CTG ATG ATC GGG CTC ATC TGG AGG TCC ATC CAC

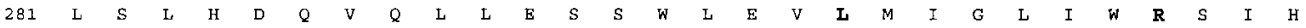
925 TGC CCC GGC AAA CTC ATC TTC GCA CAG GAC CTC ATA CTG GAC AGG AGT GAG GGC GAC TGT GTT GAA GGC ATG GCC GAG ATC TTC

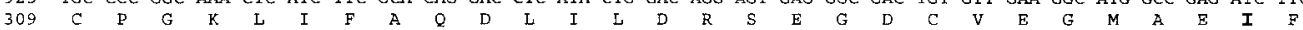
1009 GAC ATG CTG CTT GCA ACT GCC TCC CGT TTC CGC ATG CTC AAA CTC AAA CCT GAG GAG TTT GTC TGC CTC AAA GCT ATC ATC CTG

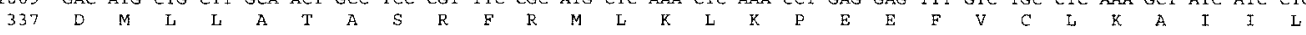
1093 CTC AAC TCT GGP GCC TTC TCT TTT TGC ACT GGC ACA ATG GAG CCC CTC CAC GAC AGT GCT GCA GTG CAG AAC ATG CTC GAC ACC $\begin{array}{llllllllllllllllllllllllllllll}365 & \text { L } & N & S & G & A & F & S & F & C & T & G & T & M & \text { B } & \text { P } & \text { L } & \text { H } & \text { D } & S & A & A & V & Q & N & M & \text { L } & \text { D } & \text { T }\end{array}$ 1177 ATC ACC GAC GCT CTC ATA CAT CAC ATC AAC CAA TCT GGA TGC TCG GCT CAG CAG CAG tCG AGA CGg CAG GCC CAG CTG CTC CTC

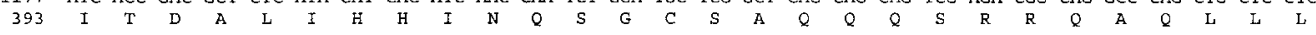

1261 CTG CTC TCC CAC ATC AGA CAC ATG AGC AAC AAA GGC ATG GAG CAT CTC TAC AGC ATG AAG TGC AAG AAC AAG GTG CCT CTG TAC

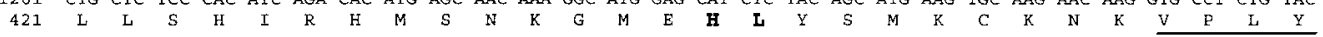
1345 GAC CTG CTG CTG GAG ATG CTG GAC GCT CAC CGC GTC CAC CGC CCC GAC AGA CCA GCT GAG ACC TGG TCC CAC GCT GAC AGA GAG

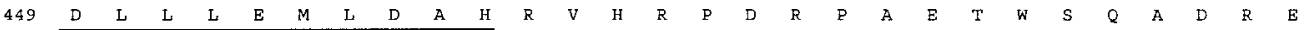
1429 CCT CTC TTC ACC TCC AGA AAC AGC AGC AGC AGC AGC GGT GGT GGT GGT GGA GGC TCC TCA TCA GCT GGC TCC ACT TCA GGA CCA

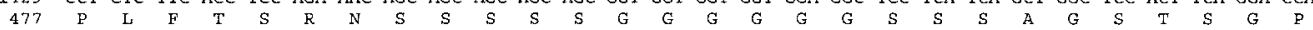

1513 CAG GTC AAC CTT GAG AGC CCC ACA GGT CCC GGC GTC CTG CAG CTC CGA GTG CAC CCA CAT CCT ATG AAA CCT ACA GAA TGA AAGC

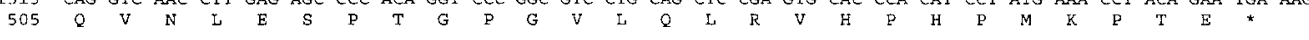

1598 TAAAGGTTGTATAATAATTCATTTGAAGAGATAATTATITATGAATTATGTGATTTTTGTAGCTGTAGTTGTTTAGGGAGACATTTTTCCTTTGCACTACTCCGGTTCACGT 1710 CAATACGAGCTTCAGCACAGTTAATCTTCTGCGCACGCINTPCAGAAAATCTGTGATTTCGAGCTTTACAATACAGCTTCTTATTTCCAGGTGTTAGTGTATATTGTGGCAC 1822 TCTGTCAGCTACAGTGATTGGAAATGACGAGCAGCTAATTTTTTGTGTGTTTTTGCTTCAACCAAAGTGCACTTCCTCTTGGGTTTAAGGGGCTGTTGGGCATTATTTTTAC 1934 TTCTAAATATAACGATGATAAAACTGGTTAATAAAATGGATGTTTGAGGACTGACTGCCAGGTATTTTTAATTTGATATACTTGGTGAACAGATAGTTAATTAATGAGATTA 2046 TGAAATGAAGAGCATCAAGGATTTATCTGTTGAATTATGAGTAACTAAAAATTGCACAATCAAATCCAAGAGGTGAAGGAAGCCAAGCTTTTATGTTGGGTAACTTCAGCCT

2158 GTCTTTAGCTTTTTGTGTGTGTAGTCGGT"ITGTTCTTCATGCTTACCTGCACCAAAGGTTTTTTTTPAATMTGITACATCATAATTGTATGTGTGTGTGTGTGTGTGTGTG

2270 TGTGTGAGTATCAGAGAGACAGAGAGAAATGTGAGAAAGAGATTTTTGCCTTAACGAAGATTTCAAGCATCAAATGACCGGTTACTATTCATCCACTCATTCAGTGATTACA

2382 TGTTGAGTTTGGCTGGATTTACACACCTCCAAAAACCCTAATTTGTCATAGTGCGCATTCATATATGCCGTTGT"T"TTATTTCTCTTAAACTACGCCAAATTTGATTCATTC 2494 ATCAGTAAGTCTAAACATGAGCTGGTGTTTTCTTTATTAGCTGCTAACTTGCTAGTGTCGTAGTTTAGTTTATAAAATATCCAAAGGAAAAAAAAGGCACAAAGGTGACAT

2605 TTAAATAACCTTTTTGCTTCCGACAAACAATGTAAATGAAGAAGGTATTAACATTTTATAATCAAAAATGACTACTGTAAAAACAAAAATATTGAATCTGAATCTGAATTAAT

2718 TGCTAAAAATCTAAAATGTTATCCTTTAATTGGGGTGITGTGATTTTGATGATAATCGCGATATCAAGAAATTGTCATGATTACAAATGAAAAACAAAAGCTGAAAATCTT

2830 CAAGGTGTETTCAGATAGGAGCGAAGCAAATGTTIGTTTTGGCCAACATCTTCTTCTTTGGGGACATTTAAAATGTTTTATTTTAATTCTTTATCATAAAAAAAAGACGAT

2942 CGTAAAATAACAATAAACCAGACTAGAGATTCATCCGCGIGCCTTAGGTTAAGATTATTAAGCCTAGCTCACTGTGATCAGAAGATGCTGCTTTTAATTGAAGCCACTTGTT

3054 CCTTGTTTGATGATACTGTGTCTGCACATGGTTGCGCACCACAGATTTAGAGCAGGTCAAAAAATTGGAGTAACT2TGTTGAAGCAGAAAACTTTCAGTTTGAACTTGGGTA

3166 ARTATATTGAAAGAAGGGTCAACAGTTGACTGACATGTAGTGTTTGCAGGCAGTTATGTTGACAGAATATAAACGATAAACAAAAAGATGCAATTACAGCCGAGTCTCATCC

3278 TCACGCAGGATTCAAAGATTGGGCTCAGCCACTTGAGCTCTGCTGTGTGATTCCAGTCAAACCTGATCTGACTGTCGTTTGTTTACTGTGCTTAAAAAAATAAAAGCATTAA

3390 TTGAGAAAAAAAAAAAAAAAAAAAAAA

Figure 1 Nucleotide and deduced amino acid sequence of clone Z22 isolated from a seabream liver cDNA library (GenBank accession number AF136979). The eight cysteines of the DBD are circled and the residues corresponding to the D- and P-box are inside a rectangle. In the LBD, the region corresponding to AF2 is underlined and the amino acids recognized to be involved in $E_{2}$ binding are in bold. Sequences of specific primers used for RT-PCR are double-underlined. 
CTC CTC CAG CTC CAG AAG GTG GAC TCC AGT CGA GTT ATT CTC TCC CCG GTC CTC AGC TCC CCT ATG GAA ACC AAC CAG CCC ATC

406 TGC ATC CCC TCC CCT TAC ACC GAC CGT GGC CAC GAC TTC CCC ACC ATA CCT TTC TAC AGT GCA ACT AAT TTC AGC TAT GCC AAT

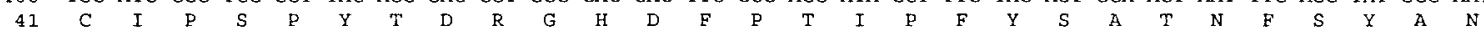

490 CCG CCG GCC ATT TCA GAC CGC CCC TCT GTC CAT CAG ACA CTA AGC CCC TCC TTA TTT TGG CCC AGC CAT GGC CAT GTG GGG ACC

$\begin{array}{llllllllllllllllllllllllllllllllll}69 & \mathrm{P} & \mathrm{P} & \mathrm{A} & \mathrm{I} & \mathrm{S} & \mathrm{D} & \mathrm{R} & \mathrm{P} & \mathrm{S} & \mathrm{V} & \mathrm{H} & \mathrm{Q} & \mathrm{T} & \mathrm{L} & \mathrm{S} & \mathrm{P} & \mathrm{S} & \mathrm{L} & \mathrm{F} & \mathrm{W} & \mathrm{P} & \mathrm{S} & \mathrm{H} & \mathrm{G} & \mathrm{H} & \mathrm{V} & \mathrm{G} & \mathrm{T}\end{array}$

574 ACC TTA CCC CTG CAC CAC CTC CAG GCT CGA CCT CAG CAC GGG CAG GCG GTT CAG AGT CCA TGG GTG GAG CTG TCG CCA CTG GAC

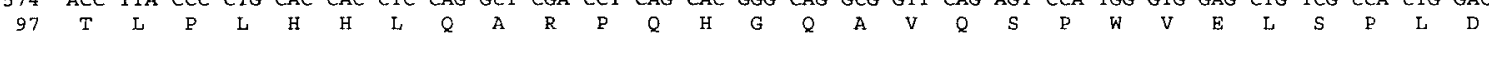

658 AAT GTG TTA ACA AGC AGT AAG AGT GCA AGG AGG CGT TCT CAG GAG AAC GAG GAG GGT GAG GTG TCA TCG GGC GGG AAG GCG GAC

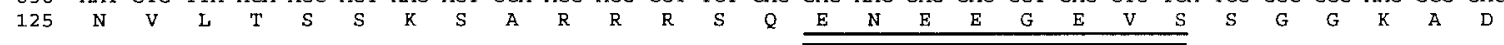

742 CTC CAC TTC TGT GCT GTG TGC CAC GAC TAC GCC TCA GGC TAC CAC TAC GGC GTC TGG TCG TGT GAG GGG TGT AAG GCC TTC TIC

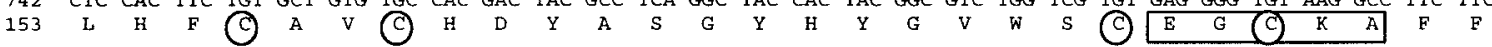

826 AAG AGG AGC ATC CAA AGA CAC AAC GAC TAC ATC TGC CCA GCA ACC AAT CAA TGC ACT ATA GAC AAG AAC CGC CGT AAG AGC TGC

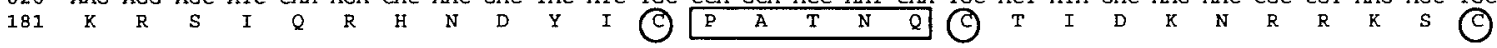

910 CAG GCG TGT CGC CTT CAC AAA TGC TAC AAC GTT GGC ATG ACC AAG TGT GGA ATG CGA AAG GAA CGT GGA AAC TTC AGG GAC CCC

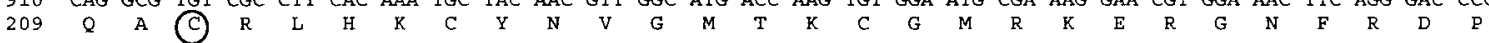

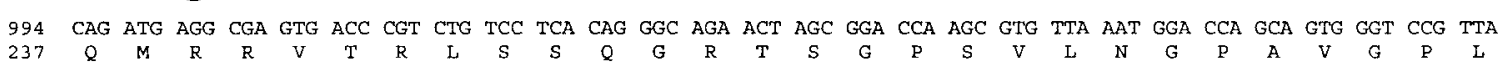

1078 AAC ACA CCC CAA CCT CCC GCA CTG ACT TCA AAG CAG CTG ATT GAG CGG ATT ATG GAG GCA GAG CCG CCA GAG ATC TAC CTC ATG

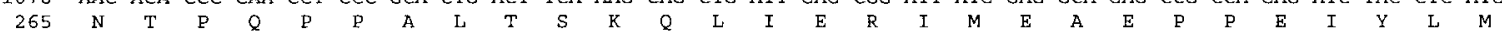

1162 AAG GAC ATG AGg AGG CCG CTG ACT GAA GCA AAC ATC ATG ATG TCG CTC ACC AAC CTG GCC GAT AAG GAG CTG GTT CAC ATG ATC

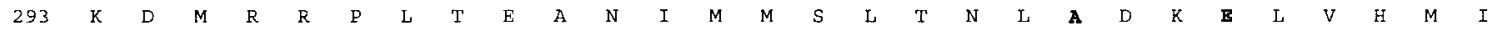

1246 ACC TGG GCC AAg AAG ATt CCA GGg TTT TTA GAG CTC GGC CTC TTG GAC CAg GTG CAC CTG TTG GAG TGC TGC tGg CTG GAg GTG

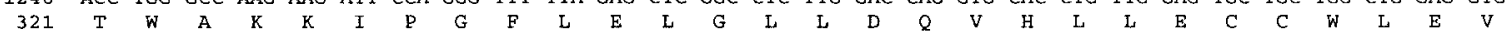

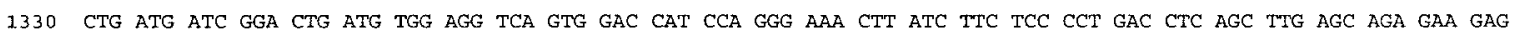

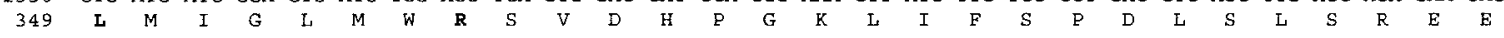

1414 GGG AGC TGT GTC CAG GGC TTC TTG GAG ATC TTT GAT ATG CTG ATA GCC GCC ACA TCC AGG GTG AGA GAG CTC AAg CTC CAG AGG

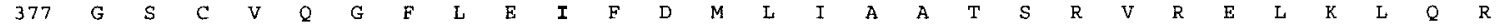

1498 GAG GAG TAC GTC TGC CTC AAG GCC ATG ATC CTC CTT AAC TCC AAC ATG TGC CTC AGC TCC TCA GAG GGC AGC GAG GAG CTG CAG

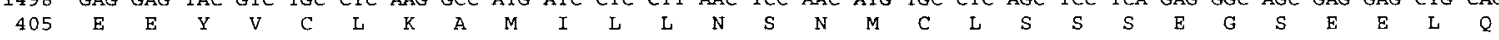

1582 AGT CGC TCC AAG CTG CTG CGT CTT CTG GAC GCC GTC ACG GAC GCT CTT GTG TGG GCC ATC GCC AAA ACC GGC CTC ACT TTC CGC

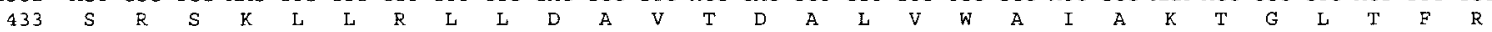

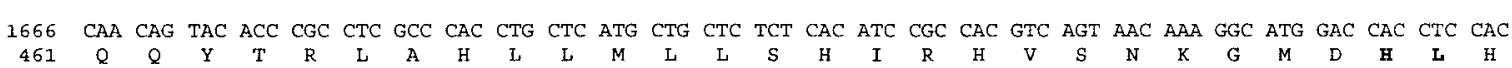

1750 GGC ATG AAA ATG AAG AAC ATG GTG CCG TTG TAT GAC CTG CTG CTG GAG ATG CTG GAC GCC CAT ATC ATG CAC AGC TCC CGT CTG

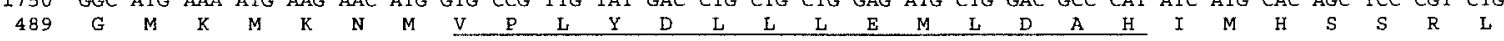

1834 CCT CGC CGG TCA CCC CAG CAG GAG ACC GTG GAA CAG TGC GAC GCT CCT GCC CGG CCA CAC AGC CCC GGT ACC TCC GGC CCC ACG

$\begin{array}{lllllllllllllllllllllllllllllll}517 & \text { P } & \text { R } & \text { R } & \text { S } & \text { P } & \text { Q } & \text { Q } & \text { E } & \text { T } & \text { V } & \text { E } & \text { Q } & \text { C } & \text { D } & \text { A } & \text { P } & \text { A } & \text { R } & \text { P } & \text { H } & \text { S } & \text { P } & \text { G } & \text { T } & \text { S } & \text { G } & \text { P } & \text { T }\end{array}$

1918 AAC ACC TGG ACT CCC AGC TGC ACC GGA GGC AGA GGT GAA CCG CAG TAg CCGGATCAGAatTCAGATGCAATGACTTTTCACGCTTTACACAAGACT

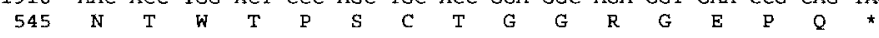

2014 AGTTCACTGCGGAGCGCTGCTTTCTTTGAACTCTCACTITGACACACCGTGCACTITCAGTTCTTCAAATTTCACTCTGCAGACAGACCAACCTAGCAGTATTCATCGGCTT

2126 TCCACCACATATTAAAAAGCCAGAGTGGATCAGGAACAAAAAAAAAAAAAAAAAA

Figure 2 Nucleotide and deduced amino acid sequence of clone Q45 isolated from seabream ovary cDNA library (GenBank accession number AF136980). The eight cysteines of the DBD are circled and the residues corresponding to the D- and P-box are inside a rectangle. In the LBD, the region corresponding to AF2 is underlined and the amino acids recognized to be involved in $E_{2}$ binding are in bold. Sequence of specific primers used for RT-PCR are double-underlined.

close to the $61 \mathrm{kDa}$ band of the luciferase positive control (not shown), thus confirming the predicted ORF. The existence of two translation products suggests that the two ATG start codons at nucleotides 286 and 384 of clone Q45 were being used.

\section{Sequence analysis}

Multisequence analysis of clones Q45 and Z22 with those of other fish and tetrapod ERs allowed identification of conserved features: the eight cysteine residues in the two zinc finger motifs common to all nuclear receptors 
Table 1 Comparison of clone Z22 and clone Q45 proteins with other species' ERs (see Materials and Methods section for sequence references and abbreviations). Overall and domain percentages of amino acid identities are indicated but, since clone Z22 was truncated in the A/B domain, amino acids corresponding to the truncated region were excluded from the analysis. The total number of amino acids or the number of residues per domain are indicated in brackets

Clone

Species/Domain

\section{$\operatorname{sbER} \alpha 2$}

rsER

$n$ tER $\alpha$

tER

KER

rtER $\alpha$

rtER $\alpha$ s

CCER

hER $\alpha$

sER

$r E R \alpha$

CER

zfER

$x$ ER

mER $\beta$

rER $\beta$

hER $\beta$

jqER $\beta$

ntER $\beta$

gfER $\beta$

eER

Clone Q45 (sbER $\beta$ )
Z22 (sbERa1)

\begin{tabular}{|c|c|c|c|c|c|}
\hline Overall & $\mathrm{A} / \mathrm{B}$ & $\mathrm{C}$ & $\mathrm{D}$ & E & $\mathrm{F}$ \\
\hline 99 (579) & 94 (137) & $100(81)$ & $100(43)$ & $100(251)$ & $100(67)$ \\
\hline 93 (581) & 94 (139) & $98(81)$ & $97(43)$ & 97 (251) & $70(67)$ \\
\hline 79 (585) & 77 (133) & $95(81)$ & $53(43)$ & 91 (251) & $44(77)$ \\
\hline 77 (583) & 76 (133) & $95(81)$ & $40(42)$ & 89 (250) & $41(77)$ \\
\hline 77 (620) & 76 (180) & $96(81)$ & $50(44)$ & $90(251)$ & $31(64)$ \\
\hline $70(622)$ & 55 (187) & $90(82)$ & $57(45)$ & $85(251)$ & $22(57)$ \\
\hline $70(577)$ & $55(142)$ & $90(82)$ & $57(45)$ & $85(251)$ & $22(67)$ \\
\hline 61 (581) & 37 (145) & $91(82)$ & $17(43)$ & $79(251)$ & $10(60)$ \\
\hline 48 (595) & 20 (179) & $90(83)$ & 13 (39) & $62(251)$ & $7(43)$ \\
\hline $48(596)$ & 23 (180) & $90(83)$ & 11 (39) & $62(251)$ & $7(43)$ \\
\hline 48 (600) & 25 (184) & $90(83)$ & $8(39)$ & $61(251)$ & $4(43)$ \\
\hline 48 (589) & $22(173)$ & $90(83)$ & $8(39)$ & $63(251)$ & $10(43)$ \\
\hline 48 (587) & $22(171)$ & $90(83)$ & 13 (39) & 63 (251) & $11(43)$ \\
\hline $48(586)$ & $23(174)$ & $91(83)$ & $8(36)$ & $61(251)$ & $8(42)$ \\
\hline 42 (549) & $16(162)$ & $83(83)$ & $8(29)$ & 57 (247) & $5(28)$ \\
\hline 42 (549) & $16(162)$ & $81(83)$ & $8(29)$ & $57(247)$ & $2(28)$ \\
\hline $42(530)$ & $16(143)$ & $83(83)$ & $9(29)$ & $57(247)$ & $7(28)$ \\
\hline $42(472)$ & 15 (99) & $84(83)$ & $11(26)$ & $57(246)$ & $2(18)$ \\
\hline 42 (557) & 16 (149) & 77 (89) & $9(25)$ & $56(249)$ & $13(45)$ \\
\hline $41(568)$ & 15 (164) & $78(91)$ & $8(28)$ & 55 (249) & $8(36)$ \\
\hline $41(573)$ & $20(165)$ & 78 (89) & $11(28)$ & 55 (249) & $8(42)$ \\
\hline 41 (559) & $14(50)$ & 75 (89) & $9(25)$ & $54(249)$ & $10(46)$ \\
\hline
\end{tabular}

Q45 (sbERß)

\begin{tabular}{|c|c|c|c|c|c|}
\hline Overall & $\mathrm{A} / \mathrm{B}$ & $\mathrm{C}$ & $\mathrm{D}$ & E & $\mathrm{F}$ \\
\hline 36 & 10 & 75 & 9 & 54 & 10 \\
\hline 36 & 10 & 76 & 11 & 54 & 8 \\
\hline 37 & 8 & 78 & 6 & 56 & 6 \\
\hline 36 & 8 & 77 & 9 & 55 & 6 \\
\hline 36 & 7 & 75 & 11 & 54 & 11 \\
\hline 36 & 8 & 71 & 6 & 54 & 4 \\
\hline 36 & 7 & 71 & 6 & 54 & 4 \\
\hline 36 & 8 & 74 & 4 & 52 & 7 \\
\hline 37 & 9 & 74 & 10 & 56 & 5 \\
\hline 38 & 9 & 74 & 10 & 56 & 0 \\
\hline 37 & 9 & 74 & 7 & 56 & 5 \\
\hline 38 & 12 & 74 & 5 & 56 & 7 \\
\hline 39 & 12 & 74 & 10 & 56 & 5 \\
\hline 38 & 14 & 74 & 5 & 55 & 2 \\
\hline 50 & 13 & 79 & 8 & 63 & 6 \\
\hline 50 & 12 & 78 & 8 & 64 & 4 \\
\hline 47 & 19 & 79 & 8 & 63 & 6 \\
\hline 46 & 16 & 79 & 7 & 63 & 8 \\
\hline 77 & 62 & 89 & 60 & 89 & 47 \\
\hline 64 & 41 & 76 & 17 & 83 & 19 \\
\hline 58 & 38 & 82 & 10 & 77 & 10 \\
\hline
\end{tabular}

(Schwabe et al. 1990); the D-box (EGCKA) and P-box (PATNQ), which have been recognized to be involved in binding to estrogen response elements (ERE) sequences (Koike et al. 1987); the ligand-dependent transactivation function (AF2) localized in the LBD (Danielian et al. 1992) is completely conserved in both clones (Figs 1 and 2); in addition, amino acids in the LBD shown in hER $\alpha$ to be involved in $\mathrm{E}_{2}$ binding (Brzozowski et al. 1997) are also conserved in sea bream ERs (Figs 1 and 2). All receptor sequences (alpha and beta) shared 60 identical amino acids in the DBD and 86 in the LBD, in no other domain did this occur.

Comparison of amino acid sequence identities between the various ERs (Table 1) showed that clone Z22 was most similar to a recently cloned seabream ER (99\%, Munoz-Cueto et al. 1999) and to most fish ERs (61-93\%), and less to eER $\alpha$, gfER $\beta$, ntER $\beta$ and clone Q45 (41$42 \%)$. Identity to tetrapod ER was $48 \%$ and to tetrapod ER $\beta 42 \%$. In contrast, clone Q45 showed $58-77 \%$ amino acid sequence identity to eER, gfER $\beta$ and $n t E R \beta$, and only $36-37 \%$ to other fish ERs. Identity to tetrapod ER $\beta$ was $47-50 \%$ and to tetrapod ER $\alpha 37-39 \%$. In both sbERs domain $\mathrm{C}$, followed by domain $\mathrm{E}$, shared the highest amino acid sequence identity with other ERs (see Table 1), and sequence conservation was much lower and diminished sequentially from domains $\mathrm{A} / \mathrm{B}, \mathrm{D}$ and $\mathrm{F}$.
Phylogeny analysis to determine the relationship between the various estrogen receptors was carried out using either the deduced whole receptor protein sequences or the various domains separately. A consensus tree with corresponding bootstrap values (from sampling 1000 trees) obtained by parsimony analysis (PAR) for the whole receptor sequence is shown in Fig. 3. This analysis produced four major groups consisting of fish and tetrapod receptor subtypes. With Neighbor Joining analysis (NJ) similar groupings were produced. A clear separation into four clades with maximum bootstrap percentages was obtained for the E domain with both PAR and NJ analysis. Analysis of the $\mathrm{C}$ domain originated three clades with PAR (placing tetrapod ER $\beta$ and clone Q45, eER and $n t E R \beta$ in the same group) and two clades with NJ (placing clone $\mathrm{Q} 45$, eER and ntER $\beta$ with tetrapod ER $\alpha$ and tetrapod ER $\beta$ with the remaining fish ERs). Analysis of $\mathrm{D}$ domain yielded inconsistent results: PAR yielded similar clustering to that of the $\mathrm{C}$ domain but NJ yielded no clear separations. No significant clustering was obtained for the $\mathrm{A} / \mathrm{B}$ and $\mathrm{F}$ domains with PAR or NJ.

Sequence identities and phylogeny analysis indicate that the two clones are closely related to identified fish ERs, and that clone $\mathrm{Z} 22$ is related to tetrapod ER $\alpha$ and clone $\mathrm{Q} 45$ is related to tetrapod ER $\beta$. However, considering 


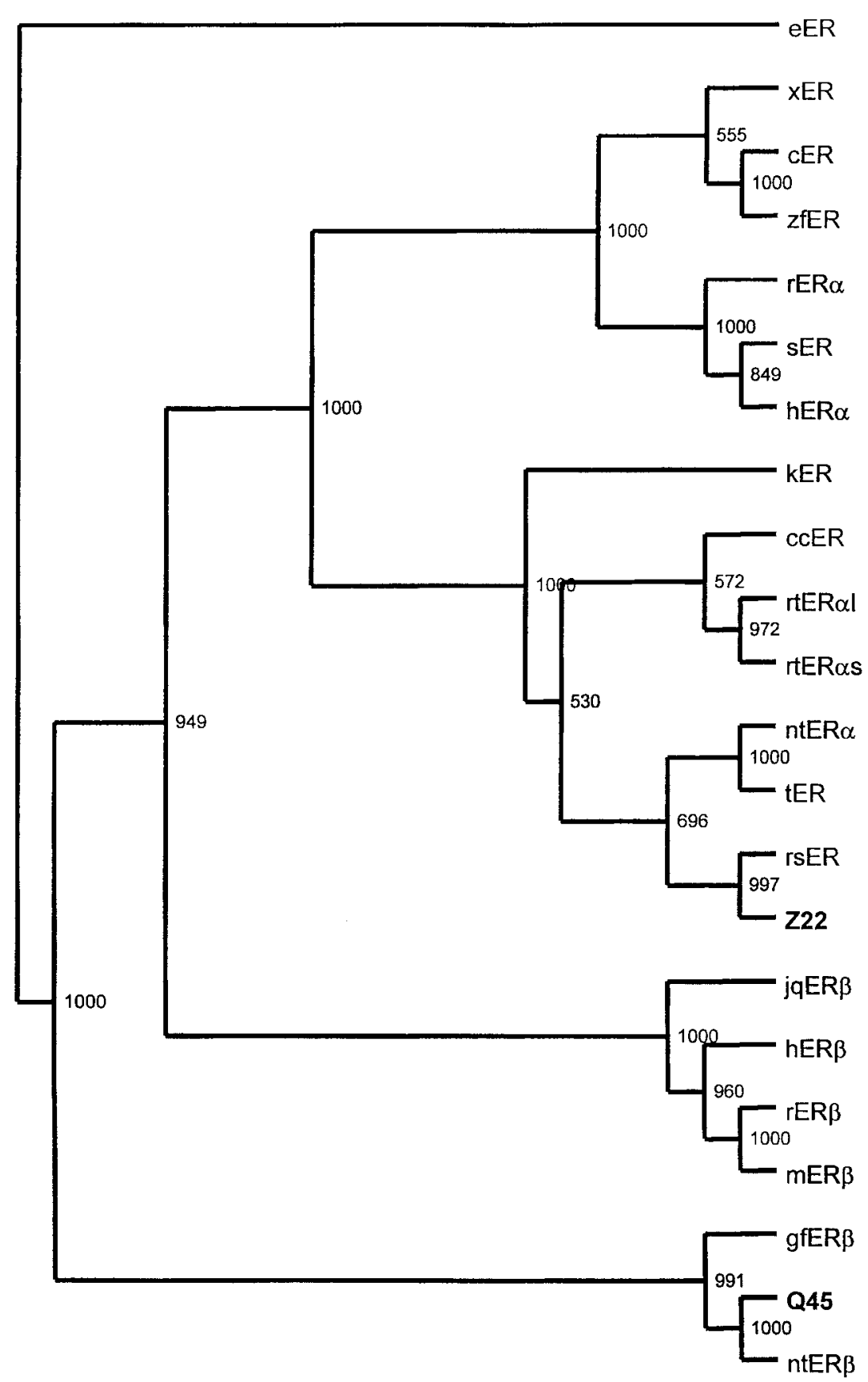

Figure 3 Phylogenetic unrooted tree - the most parsimonious consensus tree of estrogen receptors. The numbers at the forks indicate the number of times the group consisting of the species which are to the right of that fork occurred among the trees, out of 1000 trees.

the generally low sequence identities between the fish and tetrapod estrogen receptors and the wide branching pattern of the phylogenetic trees, a more detailed analysis of amino acid conservation between the various receptor sequences was carried out. On the basis of the results of this analysis and those of the phylogenetic study clone Z22 and the related fish ERs have been assigned to a group denominated fish ER $\alpha$ and clone Q45 and related fish ERs to a group designated fish ER $\beta$ and will be referred to as $\operatorname{sbER} \alpha$ and $\operatorname{sbER} \beta$ respectively. 
Table 2 Number of strictly conserved amino acids in ER within and between groups created on the basis of phylogenetic analysis. The average number of amino acids within each group are given in parentheses

\begin{tabular}{|c|c|c|c|c|}
\hline & $\begin{array}{l}\text { ERa } \\
\text { Tetrapod }\end{array}$ & $\begin{array}{l}\text { ER } \beta \\
\text { Tetrapod }\end{array}$ & $\begin{array}{l}\text { ERo } \\
\text { Fish }\end{array}$ & $\begin{array}{l}\text { ER } \beta \\
\text { Fish }\end{array}$ \\
\hline ER $\alpha$ Tetrapod (595) & 66 & & & \\
\hline ER $\beta$ Tetrapod (549) & 0 & 55 & & \\
\hline ER $\alpha$ Fish (580) & 18 & 2 & 28 & \\
\hline ERß Fish (560) & 6 & 17 & 0 & 29 \\
\hline
\end{tabular}

The amino acid conservation contrasts (i.e. amino acids that are uniquely conserved within a group and do not appear at that position in any sequence outside that group (Nicholas et al. 1997)) for the two types of fish receptors and the tetrapod ER $\alpha$ and ER $\beta$ are shown in Table 2. The number of amino acids of $\operatorname{ER} \alpha$ and $\operatorname{ER} \beta$ that are exclusively conserved in the tetrapods is proportional to the size of the ERs, suggesting similar evolutionary rates for the two receptor types. The levels of conservation contrasts found for the two types of receptors in teleosts are half those of the tetrapods and may reflect faster evolutionary rates. Conservation contrast between $\alpha$ and $\beta$ types of ER in fish and tetrapods is very low or absent (0-6 amino acids), but is high between tetrapod $\operatorname{ER} \alpha$ and fish ER $\alpha$ (18 amino acids) and between tetrapod ER $\beta$ and fish ER $\beta$ (17 amino acids). The analysis of conservation contrasts unequivocally showed that fish ERs are related to tetrapod $\alpha$ and $\beta$ and proved to be much more sensitive than a simple comparison of sequence identities to relate fish ERs to existing types in tetrapods. Uniquely conserved amino acids for each of the tetrapod and fish ER types are found mainly in the A/B (32-48\%) and E (36-67\%) domains. Uniquely conserved amino acids within the ER $\alpha$ and in ER $\beta$ groups are found mainly in the $\mathrm{E}$ domain (61 and 65\% respectively).

Motif analysis of all the tetrapod and fish sequences using the Prosite database did not show a specific pattern for any of the fish receptor types. In addition to the general ER features described above, a highly conserved amino acid sequence RRKS, corresponding to a potential cAMP- and cGMP-dependent protein kinase phosphorylation site, is found in the $\mathrm{C}$ domain. Two highly conserved $\mathrm{N}$-myristoylation sites are also present in the $\mathrm{C}$ domain and have amino acid sequences GVWSCE and $\mathrm{GM}(\mathrm{M}, \mathrm{V}, \mathrm{T}) \mathrm{K}(\mathrm{C}, \mathrm{G}) \mathrm{G}$. In the $\mathrm{E}$ domain, a totally conserved amino acid sequence SNK, potential protein kinase $\mathrm{C}$ (PKC) phosphorylation site is present.

There were also some apparent ER type specific motifs. In the A/B domain of ER $\alpha$ a mitogen-activated protein kinase phosphorylation site with the consensus motif $\mathrm{P}-\mathrm{X}_{(1,2)}-\mathrm{SP}$ is found which is not apparent in ER $\beta$ (Fig. 4). However, in tetrapod ER $\beta$ potential mitogenactivated protein kinase (MAPK) phosphorylation sites are located downstream of the corresponding region in $\mathrm{ER} \alpha$, while in fish ER $\beta$ potential MAPK sites are located upstream, except for eER $\beta$ which has two sites and sbER $\beta$ which apparently lacks a MAPK phosphorylation site. Finally, in both fish and tetrapod ER $\alpha$ LBD, a completely conserved tyrosine kinase phosphorylation site (KGMEHLY) is present.

\section{Transcripts size of sbERa and $s b E R \beta$}

Northern blot analysis was performed to characterize the sbER mRNAs. After hybridization with a $314 \mathrm{bp}$ PCR fragment encompassing the major part of the $\mathrm{N}$-terminal region of sbER $\alpha$ two mRNA transcripts of approximately 6 and $4.5 \mathrm{~kb}$ were identified in liver and a single transcript of $4.5 \mathrm{~kb}$ was detected in ovary. Four prominent ovary mRNA transcripts of approximately $6,2 \cdot 6,0.5$ and $0.3 \mathrm{~kb}$ hybridized with the full-length sbER $\beta$ cDNA, while in liver only the $0.3 \mathrm{~kb}$ transcript was detected (Fig. 5).

\section{Tissue distribution of $s b E R a$ and $s b E R \beta$}

In order to examine the distribution of $\operatorname{sbER} \alpha$ and $\operatorname{sbER} \beta$ mRNA, the sensitive method of RT-PCR analysis was performed with $E R \alpha-$ and $\beta$-specific primers. The identity of the amplified PCR products was confirmed by cloning and subsequent sequencing. By performing RT-PCR on the same samples with $\beta$-actin primers and using this to normalize the results with primers for $\operatorname{sbER} \alpha$ and $\beta$ it was possible to obtain semi-quantitative results which demonstrated important differences in the level of expression and tissue distribution of both receptors (Fig. 6). $\operatorname{sbER} \beta$ was expressed in all tissues analyzed, except gills; high levels of expression were detected in ovary and testis and also in kidney, intestine and liver. In other tissue samples expression was much lower, although heart had a slightly stronger signal. SbER $\alpha$ was only detected in testis, liver and heart with similar levels of expression.

\section{Discussion}

Two clones were isolated from seabream cDNA libraries and both showed high homology to known estrogen receptor sequences. Clone Z22, despite being the largest of several clones obtained from the pituitary and liver cDNA libraries, was assumed truncated so that the $5^{\prime} \mathrm{UTR}$ and part of the A/B domain was missing. The deduced

\footnotetext{
Figure 4 Multiple alignment of domain A/B of estrogen receptor. Potential MAPK phosphorylation sites are shaded in light gray and casein kinase II phosphorylation sites in black (see Materials and Methods for sequence references and abbreviations).
} 


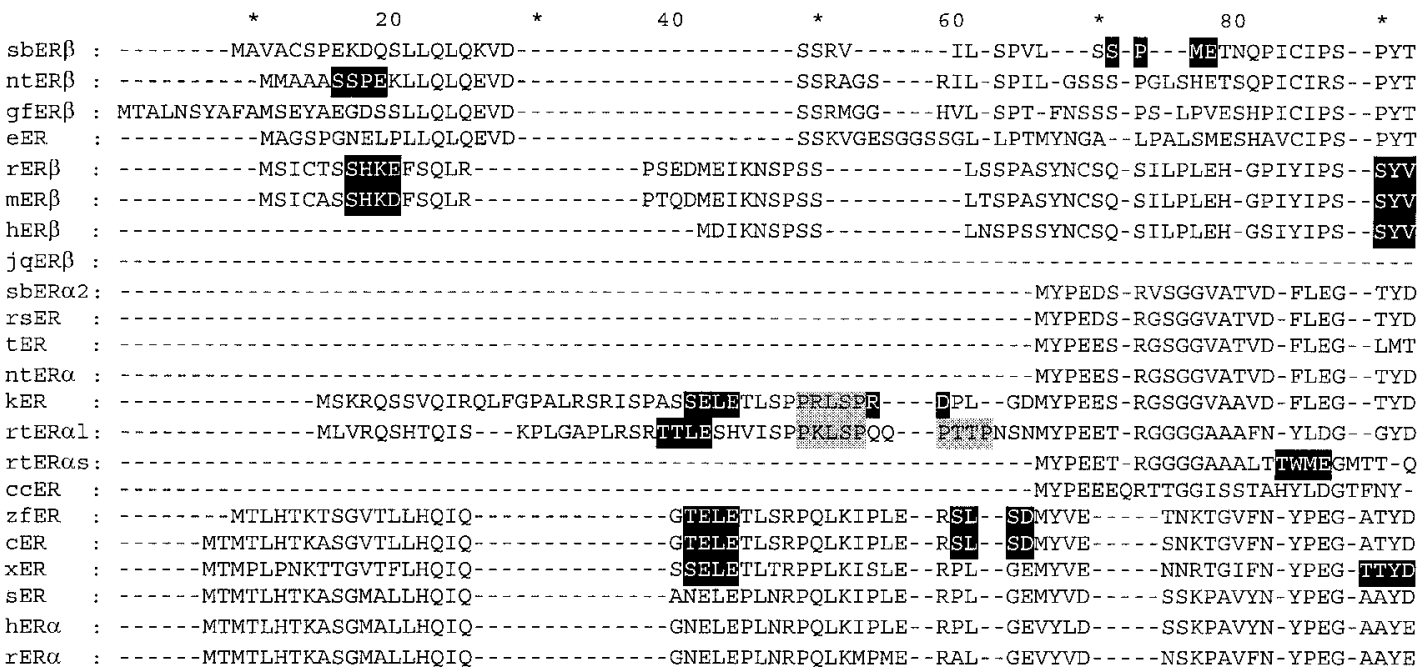

47

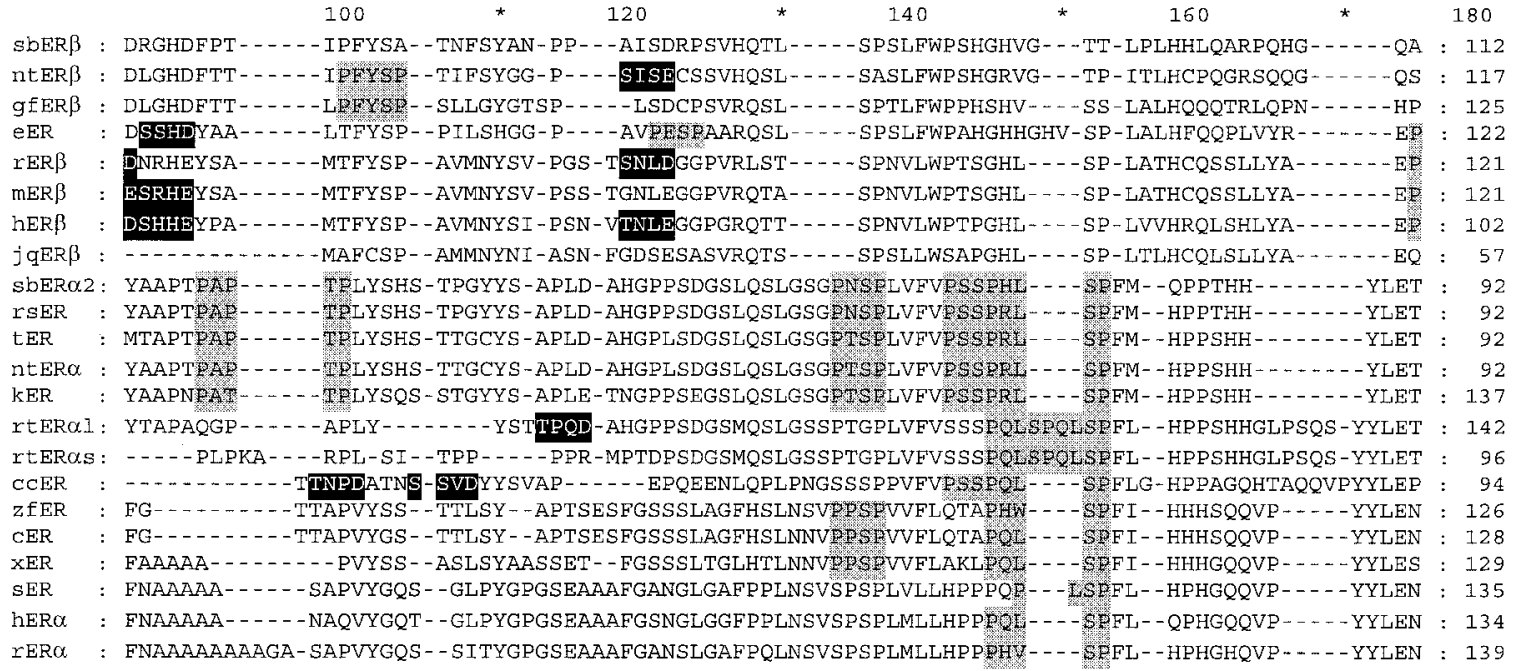

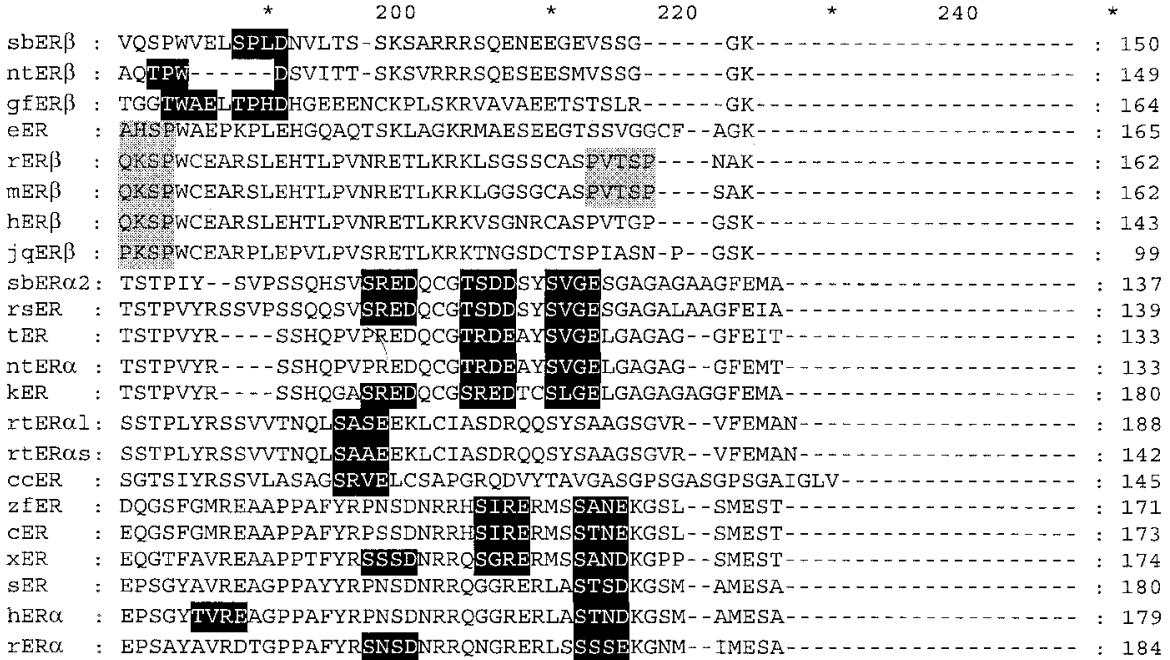




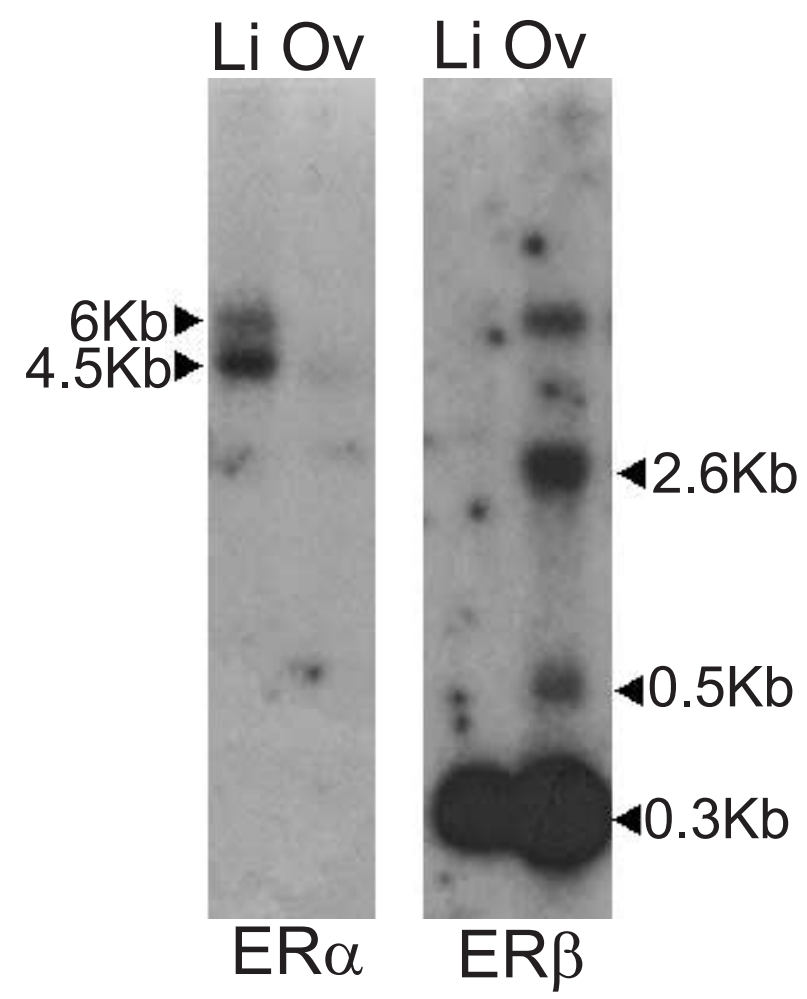

Figure 5 Northern blot analysis of seabream ER $\alpha$ and ER $\beta$. Liver (Li) and ovary (Ov) poly $(\mathrm{A})^{+}$mRNA (5 $\mu \mathrm{g}$ ) were probed with a $341 \mathrm{bp}$ cDNA fragment encoding sbER $\alpha$ and full-length sbER $\beta$.

amino acid sequence differed by only five amino acids from a recently published sbER sequence, below designated sbER $\alpha 2$ (Munoz-Cueto et al. 1999). Sequence identities (see Table 1) were also highest with other teleost ERs and ER $\alpha$ from tetrapod species. Lowest identities were found with tetrapod ER $\beta$ and the teleost eER, ntER $\beta$, gfER $\beta$ and clone Q45.

Clone $\mathrm{Q} 45$, obtained from the ovarian cDNA library, encodes a protein of 526 or 559 amino acids depending on which of two potential start codons are used. That either of the two start codons can be used was confirmed by the production of two proteins in vitro with rabbit

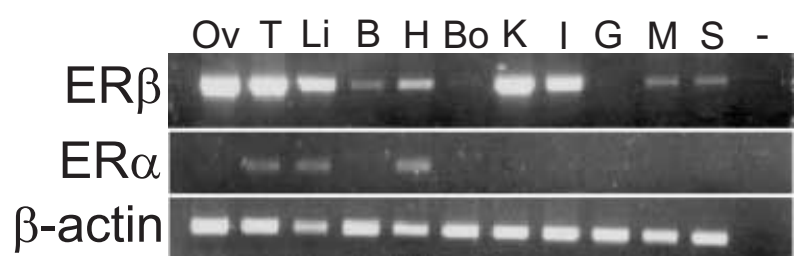

Figure 6 Tissue distribution of ER $\alpha$ and ER $\beta$ analyzed by RT-PCR. $\beta$-Actin was used as a control. Ov, ovary; T, testis; Li, liver; B, brain; $H$, heart; Bo, bone; $K$, kidney; I, intestine; $G$, gills; $M$, muscle; S, skin; -, mRNA not reversed transcribed. reticulocytes. In contrast to clone Z22, Q45 shared more identical amino acids with eER, ntER $\beta$, gfER $\beta$ and tetrapod ER $\beta$ and less with tetrapod ER $\alpha$ or with the group of teleost ERs most like Z22.

Phylogenetic analysis of the ER receptors groups them into four clusters each consisting of fish or tetrapod receptor subtypes (Fig. 3). The teleost clade consists of eER, ntER $\beta$, gfER $\beta$ and Q45 appear to be more related to tetrapod ER $\beta$, while the other fish ERs, including Z22, appear to be more related to tetrapod ER $\alpha$. This pattern of relatedness was also obtained from the analysis of the more conserved $\mathrm{C}$ and $\mathrm{E}$ domains. Further confirmation of the degree of relatedness between fish and tetrapod ERs was obtained by analysis of amino acid conservation contrasts (Table 2) among the four major clades identified by phylogenetic analysis. On the basis of these results it was concluded that clone Q45 and eER, ntER $\beta$, gfER $\beta$ were $\beta$ subtype ERs (designated sbER $\beta$ ) and clone Z22 (designated sbER $\alpha 1$ ) and the remaining fish ERs were of the $\alpha$ subtype.

The size of the deduced ER protein obtained from the various fish and tetrapod cDNA sequences is variable (Table 1). In tetrapod ER $\alpha$ it varies from 586 (Xenopus) to 600 (rat) and in fish ER $\alpha$ from 574 (rainbow trout short form) to 622 (rainbow trout long form). Tetrapod ER $\beta$ varies from 549 (mouse, rat) to 589 (zebra finch) and fish ER $\beta$ from 557 (Nile tilapia) to 573 (Japanese eel). ER $\beta$ is generally shorter than ER $\alpha$ (Table 1, see also Tchoudakova et al. 1999) although longer forms have recently been identified in mammals (Leygue et al. 1998). Some of the longer forms are derived from extra coding sequence at the $5^{\prime}$ region which is proposed to result from a single base change in transcripts upstream of the start codon causing a frame shift (Leygue et al. 1998). The length of the $\mathrm{A} / \mathrm{B}$ domain is most variable (Table 1, Fig. 4), with 133-187 amino acids in fish ER $\alpha, 171-184$ in tetrapod ER $\alpha, 149-165$ in fish ER $\beta$ and 143-162 in tetrapod ER $\beta$ (excluding the partial clones of jqER $\beta$ and sbER $\alpha 1)$. Clearly the largest differences are found in teleost ER $\alpha$ and this may be explained by the recent identification of short and long forms of $E R \alpha$ in the rainbow trout (F Pakdel, R Metivier, G Flouriot \& Y Valotaire, unpublished observations) which differ by up to 53 amino acids in the A/B domain. The cDNA for sbER $\alpha 1$ differs from sbER $\alpha 2$ (Munoz-Cueto et al. 1999) by five amino acids in the A/B domain (Fig. 4). sbER $\alpha 2$ has $\operatorname{Gln}^{83}$ (equivalent to $\mathrm{Gln}^{122}$ in hER $\alpha$ ) instead of His (present in all other ERs), Ala-Asn ${ }^{85}$ instead of Pro-Thr and lacks Arg-Ser after Tyr ${ }^{98}$, indicating that multiple variants, differing in the $\mathrm{A} / \mathrm{B}$ domain, of $\mathrm{ER} \alpha$ also occur in seabream. Two variants of $E R \alpha$ have also been identified in catfish (Xia et al. 1999). A number of ER $\alpha$ and $\beta$ variants have also been identified in other species, and in fish (e.g. Chu \& Fuller 1997, Murphy et al. 1997, Flouriot et al. 1998, Lu et al. 1998, Maruyama et al. 1998, Leygue et al. 1999), up to three variants of $\operatorname{gfER} \beta$ may 
exist (Tchoudakova et al. 1999) and four variants of tER (Tan et al. 1996).

The length of the $\mathrm{C}$ and $\mathrm{E}$ domains of all ERs has been highly conserved (ER $\alpha$ 81-83 and 250-251 amino acids; ER $\beta$ 83-91 and 246-249 amino acids). However, despite small variations, tetrapod $\mathrm{ER} \beta$ has the shortest $\mathrm{D}$ domain, 25-29 amino acids compared with 31-33 for fish ER $\beta$, 36-39 in tetrapod ER $\alpha$ and 42-45 amino acids in fish $\mathrm{ER} \alpha$. The largest $\mathrm{F}$ domains are found in the $\alpha$ receptor subtype (57-77 for fish ER $\alpha, 42-43$ for tetrapod ER $\alpha$, 36-45 for fish ER $\beta$ and 18-28 for tetrapod ER $\beta$ ) and there appears to be a trend for larger $F$ domains in teleosts, particularly in the more advanced teleosts (Table 1). The significance of these differences is not clear but it has been suggested that this domain may be important in determining the final conformation of the receptor-ligand complex, thus affecting the potential for interaction with cofactors or transcription factors in a particular cell context (Montano et al. 1995). It appears that evolutionary differences of receptor function are largely reflected in the $\mathrm{F}$ domain.

General ER features revealed by motif analysis included the nuclear receptor DNA-binding region signature with the eight cysteines constitutive of the zinc-finger motifs and the D- and P-box sequences which have been recognized to be necessary for DNA binding (Koike et al. 1987, Schwabe et al. 1990). Also completely conserved among all receptors are amino acids in domain $\mathrm{E}$ of the ligand-dependent transactivation function (Danielian et al. 1992), as well as amino acids known to be involved in $\mathrm{E}_{2}$-binding (Brzozowski et al. 1997). In domain C the complete conservation of two $\mathrm{N}$-myristoylation sites overlapping with the binding region signature potentially allows covalent addition of the C14-saturated fatty acid myristate to their $\mathrm{N}$-terminal glycine residue, which must be an important feature in DNA binding. Although in the $\mathrm{E}$ domain of all ER isolated there is a conserved potential PKC phosphorylation site, available evidence suggests that only PKC $\delta$ isoform (not PKC $\alpha$ or $\varepsilon$ ) in the AF1 region participate in the signaling pathways that lead to estrogen receptor phosphorylation (Lahooti et al. 1998).

In hER $\alpha$, five phosphorylation sites have been mapped, four of which are in the A/B domain $\left(\mathrm{Ser}^{104}, \mathrm{Ser}^{106}\right.$, $\operatorname{Ser}^{118}$ and $\operatorname{Ser}^{167}$ ). Ser ${ }^{118}$ and $\operatorname{Ser}^{167}$ are the major estrogen-inducible phosphorylation sites (Ali et al. 1993, Arnold et al. 1994, Le Goff et al. 1994). The first can be phosphorylated in vitro by MAPK (Arnold et al. 1995b, Kato et al. 1995) and the second by casein kinase II (Arnold et al. 1995a). In mER $\alpha$ the corresponding Sers identified in hER $\alpha$ are phosphorylated and two additional sites, $\operatorname{Ser}^{156}$ and $\operatorname{Ser}^{158}$, have been identified which are phosphorylated by casein kinase II (Lahooti et al. 1995). A conserved MAPK phosphorylation site consensus sequence is found in all $\operatorname{ER} \alpha$, but not in $\operatorname{ER} \beta$ (Fig. 4). However, the serine residue in mouse $\operatorname{ER} \beta$ located in the corresponding ER $\alpha$ consensus MAPK phosphorylation site can also be phosphorylated by MAPK (Tremblay et al.
1997). In sbER $\beta$, ntER $\beta$ and gfER $\beta$ the sequences corresponding to hER $\alpha \mathrm{Ser}^{118}$-Pro ${ }^{119}$ are, respectively, Thr $^{96}-$ Thr $^{97}$, Thr $^{101}-$ Pro $^{102}$ and $\mathrm{Ser}^{109}-\mathrm{Ser}^{110}$ (Figure 4). Since the replacement of Ser by Thr potentially allows phosphorylation in this position it would be of interest to know whether the mitogen-activated phosphorylation pathway is used by fish ER $\beta$ and other tetrapod ER $\beta$, or whether a ligand-independent transactivation function is absent or, if present, is activated by another mechanism.

The presence of several transcripts for both $\operatorname{sbER} \alpha$ and $\operatorname{sbER} \beta$ were demonstrated by Northern blot (Fig. 5), just as found in many other fish and mammalian species (Weiler et al. 1987, Lazennec et al. 1995, Mosselman et al. 1996, Todo et al. 1996, Tremblay et al. 1997, Tchoudakova et al. 1999). Two mRNA transcripts of $\operatorname{sbER} \alpha, 6$ and $4.5 \mathrm{~kb}$ in length, were detected in liver. In the ovary only the $4.5 \mathrm{~kb}$ transcript was detected. The $4.5 \mathrm{~kb}$ mRNA should correspond to the complete sequence of the sbER $\alpha 1$ clone isolated in the present study while the bigger transcript probably correspond to a mRNA with a longer $3^{\prime}-\mathrm{UTR}$. The transcript isolated by Munoz-Cueto et al. (1999) also from liver was a smaller $3 \mathrm{~kb}$ transcript differing in the length of $3^{\prime}$-UTR and is shorter by two amino acids in the A/B domain. It will be of interest to determine if the difference in length of the $3^{\prime}$-UTR detected between the two ER $\alpha$ forms in the seabream is a consequence of alternative splicing. In the case of $\operatorname{sbER} \beta$, at least four mRNAs $(6,2 \cdot 6,0.5$ and $0.3 \mathrm{~kb})$ were detected in seabream ovary after hybridization with the full-length ER $\beta$ cDNA. In liver only the $0.3 \mathrm{~kb}$ transcript was detected. Only the two larger transcripts can potentially generate the entire coding sequence of $\operatorname{sbER} \beta$. The significance of the smaller transcripts in seabream is uncertain and small transcripts have also been reported in eel (Todo et al. 1996), mouse (Tremblay et al. 1997) and human (Mosselman et al. 1996). Additional hybridization studies using partial probes will be necessary to characterize the nature of each mRNA.

Tissue distribution of $\operatorname{sbER} \alpha$ and $\operatorname{sbER} \beta$ (Fig. 6) was different and $\operatorname{sbER} \beta$ was widespread and had a generally higher level of expression than sbER $\alpha$. The highest expression of sbER $\beta$ was detected in ovary and testis, moderate expression was observed in kidney, intestine and liver and lower expression in brain, heart, muscle and skin. Gill was the only tissue in which no signal for $\operatorname{sbER} \beta$ could be detected. ER $\beta$ and ER $\alpha$ were co-expressed in testis, liver and heart. No signal of ER $\alpha$ was visible in any other tissues analyzed, but its presence cannot be excluded. In goldfish ER $\beta$ expression has been reported to be restricted largely to the liver, brain, ovary and testis (Tchoudakova et al. 1999).

Unfortunately, data on tissue distribution of fish ER are scarce and essentially restricted to liver and central nervous system of salmonids (Anglade et al. 1994). Both sbERs are expressed in seabream liver, although the clones isolated from liver cDNA library were of the alpha subtype. 
Whether vitellogenesis in fish is mediated by heterodimerization of the two ER subtypes, as shown for human ERs (Pettersson et al. 1997, Ogawa et al. 1998a,b), requires investigation. Another action demonstrated for estrogens in teleost fish is the positive and negative feedback on the brain-pituitary complex (Saligaut et al. 1998) and in this context the distribution of ER has been characterized (Anglade et al. 1994, Linard et al. 1996). RT-PCR analysis of seabream brain only detected ER $\beta$ but further studies will be required to completely exclude the possibility that $\mathrm{ER} \alpha$ is also present.

It is notable that in sea bream $\operatorname{ER} \beta$ was clearly expressed in ovary and testis while ER $\alpha$ was most abundant in testis. This pattern of expression may indicate, in this species, a different function for each form of sbER in male and female reproductive physiology. Recent data on $\alpha \beta E R K O$ mice clearly show that only ER $\alpha$ is required for normal testicular function. However, the presence of both ER subtypes is required for maintenance of germ and somatic cells in the postnatal ovary and their absence causes the appearance of sex reversal features including structures resembling seminiferous tubules, degeneration of granulosa cells and appearance of Sertoli-like cells (Couse et al. 1999). Whether this model can be applied to seabream reproductive physiology and natural sex reversal requires elucidation.

The significance in sea bream of the expression of ER $\beta$ in heart, bone, kidney and intestine, all known targets for estrogen action in mammals (Kuiper et al. 1997, Onoe et al. 1997), is unclear since little information exists about the effects of estrogen on these tissues in teleosts.

In conclusion, the pattern of distribution of $\operatorname{ER} \alpha$ and ER $\beta$ in seabream parallels to a great extent what has been observed in mammals and, in common with mammals, seabream also produces a range of receptor transcripts in a tissue-specific manner, consistent with the reported widespread effects of estrogen in different tissues and developmental stages.

\section{Acknowledgements}

This work was sponsored by NATO's Scientific Affairs Division in the framework of the Science for Stability Programme. Sílvia Socorro was in receipt of a grant Praxis XXI BD/9241/96.

\section{References}

Ali S, Metzger D, Bornert JM \& Chambon P 1993 Modulation of transcriptional activation by ligand-dependent phosphorylation of the human oestrogen receptor A/B region. EMBO Journal 12 1153-1160.

Altschul SF, Madden TL, Schäffer AA, Zhang J, Zhang Z, Miller W \& Lipman DJ 1997 Gapped BLAST and PSI-BLAST: a new generation of protein database search programs. Nucleic Acids Research 25 3389-3402.
Anglade I, Pakdel F, Bailhache T, Petit F, Salbert G, Jego P, Valotaire Y \& Kah O 1994 Distribution of estrogen receptor-immunoreactive cells in the brain of the rainbow trout (Oncorhynchus mykiss). Journal of Neuroendocrinology 6 573-583.

Arnold SF, Obourn JD, Jaffe H \& Notides AC 1994 Serine 167 is the major estradiol-induced phosphorylation site on the human estrogen receptor. Molecular Endocrinology 8 1208-1214.

Arnold SF, Obourn JD, Jaffe H \& Notides AC 1995a Phosphorylation of the human estrogen receptor by mitogen-activated protein kinase and casein kinase II: consequence of DNA binding. Journal of Steroid Biochemistry and Molecular Biology 55 163-172.

Arnold SF, Obourn JD, Yudt MR, Carter TH \& Notides AC 19956 in vivo and in vitro phosphorylation of the human estrogen receptor. Journal of Steroid Biochemistry and Molecular Biology 52 159-171.

Brzozowski AM, Pike AC, Dauter Z, Hubbard RE, Bonn T, Engström O, Ohman L, Greene GL, Gustafsson JA \& Carlquist M 1997 Molecular basis of agonism and antagonism in the oestrogen receptor. Nature 389 753-758.

Byers M, Kuiper GG, Gustafsson JA \& Park-Sarge OK 1997 Estrogen receptor $\beta$ mRNA expression in rat ovary: down-regulation by gonadotropins. Molecular Endocrinology 11 172-182.

Chomczynski P \& Sacchi N 1987 Single-step method of RNA isolation by acid guanidinium thiocyanate-phenol-chloroform extraction. Analytical Biochemistry 162 156-159.

Chu S \& Fuller PJ 1997 Identification of a splice variant of the rat estrogen receptor $\beta$ gene. Molecular and Cellular Endocrinology 132 195-199.

Condeça JAB \& Canario AVM 1999 The effect of estrogen on the gonads and on in vitro conversion of androstenedione to testosterone, 11-ketotestosterone and estradiol-17 $\beta$ in Sparus aurata (Teleostei, Sparidae). General and Comparative Endocrinology 116 $59-72$.

Couse JF \& Korach KS 1999 Estrogen receptor null mice: what have we learned and where will they lead us? Endocrine Reviews $\mathbf{2 0}$ 358-417.

Couse JF, Hewitt SC, Bunch DO, Sar M, Walker VR, Davis BJ \& Korach KS 1999 Postnatal sex reversal of the ovaries in mice lacking estrogen receptors $\alpha$ and $\beta$. Science 286 2328-2331.

Danielian PS, White R, Lees JA \& Parker MG 1992 Identification of a conserved region required for hormone dependent transcriptional activation by steroid hormone receptors. (Published erratum appears in EMBO Journal 199211 2366.) EMBO Journal 11 1025-1033.

Enmark E, Pelto-Huikko M, Grandien K, Lagercrantz S, Lagercrantz J, Fried G, Nordenskjold M \& Gustafsson JA 1997 Human estrogen receptor $\beta$-gene structure, chromosomal localization, and expression pattern. Journal of Clinical Endocrinology and Metabolism 82 $4258-4265$.

Felsenstein J 1989 PHYLIP - Phylogeny Inference Package (Version 3.2). Cladistics 5 164-166.

Flouriot G, Griffin C, Kenealy M, Sonntag-Buck V \& Gannon F 1998 Differentially expressed messenger RNA isoforms of the human estrogen receptor- $\alpha$ gene are generated by alternative splicing and promoter usage. Molecular Endocrinology 12 1939-1954.

Green S, Kumar V, Krust A, Walter P \& Chambon P 1986a Structural and functional domains of the estrogen receptor. Cold Spring Harbor Symposia on Quantitative Biology 51 751-758.

Green S, Walter P, Greene G, Krust A, Goffin C, Jensen E, Scrace G, Waterfield M \& Chambon P 1986b Cloning of the human oestrogen receptor cDNA. Journal of Steroid Biochemistry 24 77-83.

Happe A \& Zohar Y 1988 Self-fertilization in the protandrous hermaphrodite Sparus aurata: development of the technology. In Reproduction in Fish - Basic and Applied Aspects in Endocrinology and Genetics, pp 177-180. Eds Y Zohar \& B Breton. Les colloques de l'INRA, no. 44. Tel-Aviv, Israel: INRA: Paris.

Jacobs EC, Arnold AP \& Campagnoni AT 1996 Zebra finch estrogen receptor cDNA: cloning and mRNA expression. Journal of Steroid Biochemistry and Molecular Biology 59 135-145. 
Kato S, Endoh H, Masuhiro Y, Kitamoto T, Uchiyama S, Sasaki H, Masushige S, Gotoh Y, Nishida E, Kawashima H, Metzger D \& Chambon P 1995 Activation of the estrogen receptor through phosphorylation by mitogen-activated protein kinase. Science $\mathbf{2 7 0}$ 1491-1494

Koike S, Sakai M \& Muramatsu M 1987 Molecular cloning and characterization of rat estrogen receptor cDNA. Nucleic Acids Research 15 2499-2513.

Krege JH, Hodgin JB, Couse JF, Enmark E, Warner M, Mahler JF, Sar M, Korach KS, Gustafsson JA \& Smithies O 1998 Generation and reproductive phenotypes of mice lacking estrogen receptor $\beta$. PNAS 95 15677-15682.

Krust A, Green S, Argos P, Kumar V, Walter P, Bornert JM \& Chambon P 1986 The chicken oestrogen receptor sequence: homology with v-erbA and the human oestrogen and glucocorticoid receptors. EMBO Journal 5 891-897.

Kuiper G, Enmark E, Peltohuikko M, Nilsson S \& Gustafsson JA 1996 Cloning of a novel estrogen receptor expressed in rat prostate and ovary. PNAS 93 5925-5930.

Kuiper GG, Carlsson B, Grandien K, Enmark E, Haggblad J, Nilsson S \& Gustafsson JA 1997 Comparison of the ligand binding specificity and transcript tissue distribution of estrogen receptors $\alpha$ and $\beta$. Endocrinology 138 863-870.

Kumar V, Green S, Stack G, Berry M, Jin JR \& Chambon P 1987 Functional domains of the human estrogen receptor. Cell $\mathbf{5 1}$ 941-951.

Lahooti H, White R, Hoare SA, Rahman D, Pappin DJC \& Parker MG 1995 Identification of phosphorylation sites in the mouse oestrogen receptor. Journal of Steroid Biochemistry and Molecular Biology 55 305-313.

Lahooti H, Thorsen T \& Aakvaag A 1998 Modulation of mouse estrogen receptor transcription activity by protein kinase $\mathrm{C} \delta$. Journal of Molecular Endocrinology 20 245-259.

Lakaye B, Foidart A, Grisar T \& Balthazart J 1998 Partial cloning and distribution of estrogen receptor $\beta$ in the avian brain. Neuroreport 9 2743-2748

Laudet V 1997 Evolution of the nuclear receptor superfamily: early diversification from an ancestral orphan receptor. Journal of Molecular Endocrinology 19 207-226.

Lazennec G, Huignard H, Valotaire Y \& Kern L 1995 Characterization of the transcription start point of the trout estrogen receptor-encoding gene: evidence for alternative splicing in the $5^{\prime}$ untranslated region. Gene 166 243-247.

Le Goff P, Montano MM, Schodin DJ \& Katzenellenbogen BS 1994 Phosphorylation of the human estrogen receptor. Identification of hormone-regulated sites and examination of their influence on transcriptional activity. Journal of Biological Chemistry 269 $4458-4466$.

Leygue E, Dotzlaw H, Lu B, Glor C, Watson PH \& Murphy C 1998 Estrogen receptor beta: mine is longer than yours? Journal of Clinical Endocrinology and Metabolism 83 3754-3755.

Leygue E, Dotzlaw H, Watson PH \& Murphy LC 1999 Expression of estrogen receptor $\beta 1, \beta 2$, and $\beta 5$ messenger RNAs in human breast tissue. Cancer Research 59 1175-1179.

Linard B, Anglade I, Corio M, Navas JM, Pakdel F, Saligaut C \& Kah O 1996 Estrogen receptors are expressed in a subset of tyrosine hydroxylase-positive neurons of the anterior preoptic region in the rainbow trout. Neuroendocrinology 63 156-165.

Lu B, Leygue E, Dotzlaw H, Murphy LJ, Murphy LC \& Watson PH 1998 Estrogen receptor $\beta$ mRNA variants in human and murine tissues. Molecular and Cellular Endocrinology 138 199-203.

Madigou T, Tiffoche C, Lazennec G, Pelletier J \& Thieulant ML 1996 The sheep estrogen receptor: cloning and regulation of expression in the hypothalamo-pituitary axis. Molecular and Cellular Endocrinology 121 153-163.

Maruyama K, Endoh H, Sasaki-Iwaoka H, Kanou H, Shimaya E, Hashimoto S, Kato S \& Kawashima H 1998 A novel isoform of rat estrogen receptor $\beta$ with 18 amino acid insertion in the ligand binding domain as a putative dominant negative regulator of estrogen action. Biochemical and Biophysical Research Communications 246 142-147.

Montano MM, Müller V, Trobaugh A \& Katzenellenbogen BS 1995 The carboxy-terminal $\mathrm{F}$ domain of the human estrogen receptor: role in the transcriptional activity of the receptor and the effectiveness of antiestrogens as estrogen antagonists. Molecular Endocrinology 9 814-825.

Mosselman S, Polman J \& Dijkema R 1996 ER $\beta$ : identification and characterization of a novel human estrogen receptor. FEBS Letters 392 49-53.

Munoz-Cueto JA, Burzawa-Gerard E, Kah O, Valotaire Y \& Pakdel F 1999 Cloning and sequencing of the gilthead sea bream estrogen receptor cDNA. DNA Sequence 10 75-84.

Murphy LC, Dotzlaw H, Leygue E, Douglas D, Coutts A \& Watson PH 1997 Estrogen receptor variants and mutations. Journal of Steroid Biochemistry and Molecular Biology 62 363-372.

Nicholas KB, Nicholas HB Jr \& Deerfield DW II 1997 GeneDoc: analysis and visualization of genetic variation. EMBNEW. NEWS 4 14.

Ogawa S, Inoue S, Orimo A, Hosoi T, Ouchi Y \& Muramatsu M 1998 a Cross-inhibition of both estrogen receptor $\alpha$ and $\beta$ pathways by each dominant negative mutant. FEBS Letters 423 129-132.

Ogawa S, Inoue S, Watanabe T, Hiroi H, Orimo A, Hosoi T, Ouchi Y \& Muramatsu M 19986 The complete primary structure of human estrogen receptor $\beta(\mathrm{hER} \beta)$ and its heterodimerization with $\mathrm{ER} \alpha$ in vivo and in vitro. Biochemical and Biophysical Research Communications 243 122-126.

Onoe Y, Miyaura C, Ohta H, Nozawa S \& Suda T 1997 Expression of estrogen receptor $\beta$ in rat bone. Endocrinology 138 4509-4512.

Osterlund M, Kuiper G, Gustafsson JA \& Hurd YL 1998 Differential distribution and regulation of estrogen receptor- $\alpha$ and $-\beta$ mRNA within the female rat brain. Molecular Brain Research 54 175-180.

Pace P, Taylor J, Suntharalingam S, Coombes RC \& Ali S 1997 Human estrogen receptor $\beta$ binds DNA in a manner similar to and dimerizes with estrogen receptor $\alpha$. Journal of Biological Chemistry 272 25832-25838.

Pakdel F, Le Gac F, Le Goff P \& Valotaire Y 1990 Full-length sequence and in vitro expression of rainbow trout estrogen receptor cDNA. Molecular and Cellular Endocrinology 71 195-204.

Pettersson K, Grandien K, Kuiper GG \& Gustafsson JA 1997 Mouse estrogen receptor $\beta$ forms estrogen response element-binding heterodimers with estrogen receptor $\alpha$. Molecular Endocrinology 11 1486-1496.

Picard D, Kumar V, Chambon P \& Yamamoto KR 1990 Signal transduction by steroid hormones: nuclear localization is differentially regulated in estrogen and glucocorticoid receptors. Cell Regulation 1 291-299.

Saligaut C, Linard B, Mananos EL, Kah O, Breton B \& Govoroun M 1998 Release of pituitary gonadotrophins GtH I and GtH II in the rainbow trout (Oncorhynchus mykiss) -modulation by estradiol and catecholamines. General and Comparative Endocrinology 109 302-309.

Santos CRA, Power DM, Kille P, Llewellyn L, Ramsurn V, Wigham T \& Sweeney GE 1997 Cloning and sequencing of a full-length sea bream (Sparus aurata) $\beta$-actin cDNA. Comparative Biochemistry and Physiology 117B 185-189.

Schwabe JW, Neuhaus D \& Rhodes D 1990 Solution structure of the DNA-binding domain of the oestrogen receptor. Nature 348 458-461.

Shughrue PJ, Lane MV, Scrimo PJ \& Merchenthaler I 1998 Comparative distribution of estrogen receptor $\beta(E R \alpha)$ and $\beta$ $(E R \beta)$ mRNA in the rat pituitary, gonad, and reproductive tract. Steroids 63 498-504.

Tan NS, Lam TJ \& Ding JL 1995 Molecular cloning and sequencing of the hormone-binding domain of Oreochromis aureus estrogen receptor gene. DNA Sequence 5 359-370. 
Tan NS, Lam TJ \& Ding JL 1996 The first contiguous estrogen receptor gene from a fish, Oreochromis aureus: evidence for multiple transcripts. Molecular and Cellular Endocrinology 120 177-192.

Tchoudakova A, Pathak S \& Callard GV 1999 Molecular cloning of an estrogen receptor $\beta$ subtype from the goldfish, Carassius auratus. General and Comparative Endocrinology 113 388-400.

Thompson JD, Gibson TJ, Plewniak F, Jeanmougin F \& Higgins DG 1997 The CLUSTAL X windows interface: flexible strategies for multiple sequence alignment aided by quality analysis tools. Nucleic Acids Research 25 4876-4882.

Todo T, Adachi S \& Yamauchi K 1996 Molecular cloning and characterization of Japanese eel estrogen receptor cDNA. Molecular and Cellular Endocrinology 119 37-45.

Tora L, White J, Brou C, Tasset D, Webster N, Scheer E \& Chambon P 1989 The human estrogen receptor has two independent nonacidic transcriptional activation functions. Cell $\mathbf{5 9}$ $477-487$.

Touhata K, Kinoshita M, Toyohara H \& Sakaguchi M 1998 Sequence and expression of a cDNA encoding the red seabream estrogen receptor. Fisheries Science 64 131-135.

Tremblay GB, Tremblay A, Copeland NG, Gilbert DJ, Jenkins NA, Labrie F \& Giguere V 1997 Cloning, chromosomal localization, and functional analysis of the murine estrogen receptor $\beta$. Molecular Endocrinology 11 353-365.

Tzukerman MT, Esty A, Santiso-Mere D, Danielian P, Parker MG, Stein RB, Pike JW \& McDonnell DP 1994 Human estrogen receptor transactivational capacity is determined by both cellular and promoter context and mediated by two functionally distinct intramolecular regions. Molecular Endocrinology 8 21-30.

Walter P, Green S, Greene G, Krust A, Bornert JM, Jeltsch JM, Staub A, Jensen E, Scrace G, Waterfield M \& Chambon P 1985 Cloning of the human estrogen receptor cDNA. PNAS $\mathbf{8 2}$ 7889-7893.

Weiler IJ, Lew D \& Shapiro DJ 1987 The Xenopus laevis estrogen receptor: sequence homology with human and avian receptors and identification of multiple estrogen receptor messenger ribonucleic acids. Molecular Endocrinology 1 355-362.

Xia Z, Patiño R, Gale WL, Maule AG \& Densmore LD 1999 Cloning, in vitro expression, and novel phylogenetic classification of a channel catfish estrogen receptor. General and Comparative Endocrinology 113 360-368.

Received 20 January 2000

Accepted 29 March 2000 